\title{
Midwives' adherence to guidelines on the management of birth asphyxia in Malawi
}

\author{
Bertha Chikuse $^{1}$, Ellen Chirwa ${ }^{2}$, Alfred Maluwa ${ }^{3 *}$, Address Malata ${ }^{4}$, Jon Odland ${ }^{5}$ \\ ${ }^{1}$ Ntcheu District Hospital, Ntcheu, Malawi \\ ${ }^{2}$ Department of Maternal and Child Health, Kamuzu College of Nursing, University of Malawi, Blantyre, Malawi \\ ${ }^{3}$ Research Directorate, Kamuzu College of Nursing, University of Malawi, Lilongwe, Malawi \\ ${ }^{4}$ Department of Maternal and Child Health, Kamuzu College of Nursing, University of Malawi, Lilongwe, Malawi \\ ${ }^{5}$ Faculty of Health Sciences, University of Tromso, Norwegian Agency for Development Cooperation, Tromso, Norway \\ Email: aomaluwa@kcn.unima.mw
}

Received 14 September 2012; revised 18 October 2012; accepted 12 November 2012

\begin{abstract}
A study was conducted to determine midwives adherence to guidelines on management of birth asphyxia at Queen Elizabeth Central Hospital in Blantyre district, Malawi. The study design was descriptive cross sectional using quantitative data analysis method on 75 midwives that were working in the maternity unit of the hospital. A structured questionnaire was used to collect data on participant's demographic characteristics and midwives' comprehension of birth asphyxia and an observational check list was used to observe midwives' adherence to WHO resuscitation guidelines. In addition midwives were observed on their adherence to the Integrated Maternal and Neonatal Health guidelines that were developed by the Malawi Ministry of Health. The findings indicate that the midwives had knowledge of birth asphyxia in general. However, there were gaps in their ability to identify warning signs of birth asphyxia through partograph use. In addition the midwives did not adhere to 9 out of the 21 steps of the resuscitation guideline. Generally there was substandard adherence to guidelines on identification of warning signs of birth asphyxia and neonatal resuscitation. On the other hand, the facility did not have adequate resuscitation equipment and supplies. The results are discussed in relation to the importance of adhering to resuscitation guidelines in the management of birth asphyxia for babies that do not breathe at birth. Training of the midwives on partograph use and resuscitation to improve neonatal outcomes is recommended. It is recommended further that the health facility should have adequate resuscitation equipment and supplies.
\end{abstract}

Keywords: Birth Asphyxia; Resuscitation Guidelines;

*Corresponding author.
Neonates; Use of Partograph; Virginia Apgar Scoring

\section{INTRODUCTION}

Birth asphyxia is one of the common causes of early neonatal deaths, accounting for approximately $30 \%$ of the 5 million neonatal deaths globally each year [1] and ranks the second most important cause of neonatal deaths after neonatal infections. In addition, birth asphyxia is closely associated with major developmental sequelae such as cerebral palsy, cognitive impairment, epilepsy and chronic illness later in life [2]. The incidence of birth asphyxia is reported to be higher in the developing countries and Sub-Saharan Africa where neonatal mortality rates are as high as $40-50$ per thousand live births [1]. In Malawi, neonatal mortality is estimated at 31 per thousand live births [3] with birth asphyxia accounting for $22 \%$ of the neonatal mortality [4]. At Queen Elizabeth Central Hospital (Chatinkha Maternity), HMIS data from January to March (2010) indicated that there were 92 babies who had birth asphyxia out of 1500 total deliveries. Worldwide, studies by WHO [5] reported that substandard care in the management of obstetric complications such as obstructed labor, ruptured uterus, eclampsia, antepartum haemorrhage and absence of resuscitation were contributing factors to deaths due to birth asphyxia. However, perinatal birth asphyxia and hypothermia are conditions that are directly linked to quality of care during childbirth and are averted by the availability and use of good quality obstetric care [5].

In Malawi, the Ministry of Health has been conducting training of midwives on Saving Newborn Lives, Essential Newborn Care, Basic Emergency Obstetric Care (BEMOC) and Integrated Maternal and Neonatal Care. All these training courses have a component of resuscitation of the asphyxiated newborn. Furthermore, Ministry of Health through the Reproductive Health Unit distributed guidelines and protocols on the management of 
emergency obstetric complications, which have neonatal resuscitation of birth asphyxia as one of the components. These guidelines and protocols aim at assisting the midwives when conducting resuscitation of the asphyxiated neonates.

Despite all these efforts by the Ministry, there are still gaps in the management of birth asphyxia among midwives in most maternity departments of the health facilities in Malawi. It has been observed that midwives do not always use the guidelines. Consequently, there are still many neonatal deaths due to birth asphyxia. Studies on birth asphyxia in Malawi are limited as most studies have mostly focused on the care for the mother. Therefore the purpose of this study was to determine the midwives' adherence to guidelines on management of birth asphyxia at Chatinkha Maternity of Queen Elizabeth Central Hospital in Malawi. Specifically, the study assessed the comprehension of midwives regarding birth asphyxia; their ability to identify warning signs of birth asphyxia and their adherence to the guidelines on resuscitation procedure of the asphyxiated neonate.

\section{METHODS}

\subsection{Design}

The study utilized a descriptive cross-sectional design and quantitative data collection and analysis method.

\subsection{Setting}

The research was conducted at Chatinkha Maternity of Queen Elizabeth Central Hospital. This is a tertiary referral facility in Blantyre district located in the Southern Region of Malawi. The study period was 2 months from July to August, 2011.

\subsection{Sample}

All 75 midwives that were working in the maternity ward of the facility at the time of the study consented and participated in the study.

\subsection{Inclusion and Exclusion Criteria}

Midwives who were working at Chatinkha maternity during the study period were included in the study. Observation was made on the midwives who assisted women that delivered an asphyxiated baby. Excluded were the midwives who were not working at Chatinkha maternity, student midwives, midwives on orientation and other cadres of staff other than midwives.

\subsection{Data Collection}

A structured questionnaire was used to collect data on participants' demographic characteristics and midwives comprehension of birth asphyxia. A checklist was used to assess midwives' ability to identify warning signs of birth asphyxia and was developed from indicators on the partograph. In addition, observational checklist to determine midwives adherence to guidelines on resuscitation was adapted from the Integrated Maternal and Neonatal Care [6] and WHO guidelines [7]. Another ordinary checklist was used to assess availability of resources. Labor graphs were reviewed to see if midwives were able to recognize warning signs of birth asphyxia. The midwives filled the questionnaire on their own while the observational checklist was filled by the senior author of this study who is a State Registered Nurse-Midwife. She was assisted by two trained research assistants who were qualified Nurse-Midwife Technicians.

\subsection{Data Management and Analysis}

The data from the questionnaire and checklist were entered into SPSS version 16.0. During analysis, descriptive statistics were computed and the results are reported as frequencies and percentages.

\subsection{Ethical Consideration}

The study was approved by the College of Medicine Research and Ethics Committee (COMREC) in Malawi and institutional clearance was obtained from the Director of Queen Elizabeth Central Hospital. All participants gave informed consent before they participated in the study. All other ethical issues such as avoiding harm and maintaining confidentiality were observed.

\section{RESULTS}

\subsection{Demographic Characteristics of Participants}

The age of the midwives ranged from 20 to 59 with $25.3 \%, n=19$ of them being in the 25 - 29 age group. The younger generation (20 - 29 years old) comprised $54.7 \%, \mathrm{n}=41$ of the participants. The participants were midwives of different cadres with most of them $(65 \%, n=$ 49) being Nurse Midwife Technicians. The Registered Nurse Midwives comprised 25\%, $n=19$, and the least $10 \%(n=7)$ were Enrolled Midwives.

All the participants had professional qualifications in nursing and midwifery but at different levels. The Nurse Technicians possessed Diploma in Nursing/Midwifery, while the Registered Nurse Midwives had Degrees in Nursing/Midwifery. The enrolled nurse-midwives possessed certificates in Nursing/Midwifery. A high percentage of the participants $(61.4 \% n=46)$, had worked in the maternity for less than 2 years with $46.7 \%$ having worked for less than a year. 


\subsection{Apgar Scores of Neonates That Were Resuscitated}

Majority of the neonates that were resuscitated $(60 \%, \mathrm{n}=$ 45) had an Apgar score ranging from $2 / 10$ to $4 / 10$ and the rest $(40 \%, n=30)$ had Apgar scores that ranged from $5 / 10$ to $6 / 10$.

\subsection{Midwives Comprehension of Birth Asphyxia}

The majority of midwives $(98.7 \% \mathrm{n}=74)$ were conversant with birth asphyxia. However, only $74.7 \% \mathrm{n}=56$ were able to define birth asphyxia correctly despite the fact that a high proportion, $85.3 \%, \mathrm{n}=64$ indicated that they had been trained on birth asphyxia. For those who were trained, the majority $(64 \%, n=48)$ had been trained recently, i.e., less than a year ago. Those that had been trained the past 1 to 2 years were $33.3 \%, n=25$ and those that were trained more than 3 years ago were few $(2.7 \%, \mathrm{n}=2)$.

The most well-known cause of birth asphyxia for $75 \%$, $\mathrm{n}=57$ of the participants was prolonged labor while the other two leading causes pre-eclampsia and sedation were known by few participants $(15 \%, \mathrm{n}=11)$ and $(10 \%$, $\mathrm{n}=8$ ) respectively. Participants knew only four of all the major signs of birth asphyxia. The most commonly known cause among the participants $(81 \%, \mathrm{n}=61)$ was the absence of respiration. However only $15 \%, n=11$ of the participants mentioned gasping respirations. Diminished reflexes were known by $3 \%, n=2$ of the participants and heart beat below $100 \mathrm{~b} / \mathrm{min}$ was mentioned by the least proportion of the participants $(1.3 \%, \mathrm{n}=1)$.

The participants knew neonatal requirement for resuscitation and the majority of them $(90.7 \%, \mathrm{n}=68)$ mentioned the correct Apgar score of less than 6. However, a few that did not know $(9.3 \%, \mathrm{n}=7)$ mentioned an incorrect Apgar score of 0 .

\subsection{Midwives Ability to Identify Warning Signs of Birth Asphyxia}

Midwives scored $100 \%$ in identifying neonates requiring resuscitation, thus all the neonates requiring resuscitation were identified (Figure 1). However, participants scored less than $50 \%$ on their ability to monitor the other four indicators (fetal heart, color of liquor, caput and moulding and ability to Apgar score according to Virginia Apgar scoring Chart). The least proportion $(9.3 \%, n=7)$ of participants were able to monitor caput (Figure 1).

\subsection{Midwives Adherence to Guidelines on Resuscitation of the Neonate with Birth Asphyxia}

The steps of the resuscitation guidelines and their adherence by the Midwives are shown in Table 1. Most midwives $(97.3 \%)$ provided warmth to the neonate through radiant warmer. The guidelines on quickly clearing the airway and reassessing the baby after suctioning in order of importance $(80 \%, n=60)$ and, respirations, heart rate and color monitoring $(80 \%, n=60)$ were adhered to by most of the participants (Table 1). The steps least adhered to were: If newborn starts crying, stop ventilating and continue observing breathing for 15 minutes $(12 \%, \mathrm{n}=$ $3)$, If the newborn is not breathing or breathing is slow ( $<30$ breaths per minute) or weak or severe in drawing continue ventilating until spontaneous breathing begins (15\%, $\mathrm{n}=4$ ) and after ventilating for 1 minute, stop and quickly assess if newborn is having spontaneous breathing $(21 \%$, $\mathrm{n}=5$ ) Table 1.

\section{DISCUSSION}

\subsection{Demographic Characteristics}

Chatinkha Maternity is managed by professional mid-

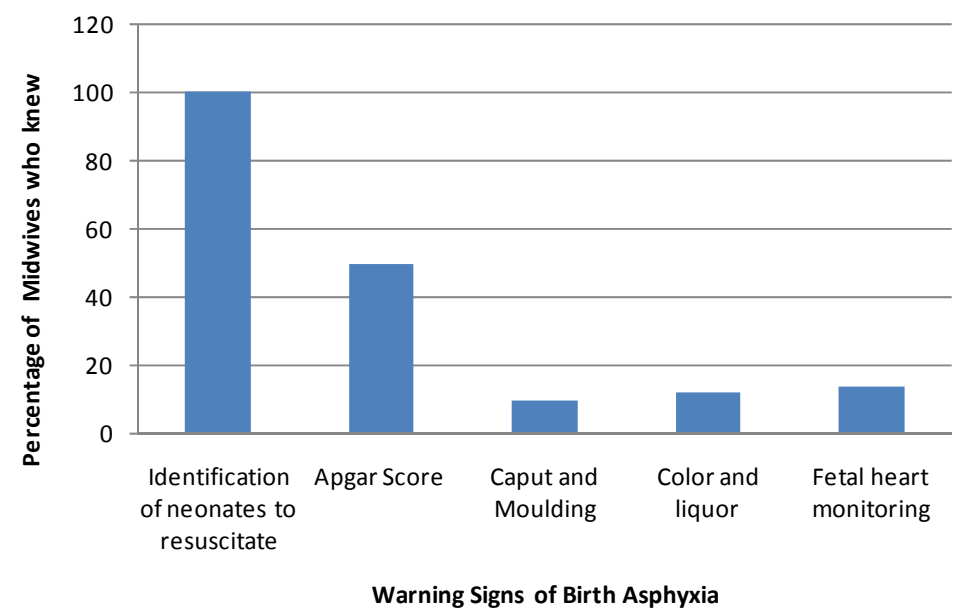

Figure 1. Midwives' ability to identify warning signs of birth asphyxia at queen elizabeth central hospital. 
Table 1. Proportions of midwives that adhered to guidelines on resuscitation of the neonate with birth asphyxia during neonatal resuscitation at Queen Elizabeth central hospital in Malawi.

\begin{tabular}{|c|c|c|}
\hline $\begin{array}{l}\text { Guideline } \\
\text { Step }\end{array}$ & Guideline & $\begin{array}{l}\text { Adherence } \\
\text { (\%) }\end{array}$ \\
\hline 1 & Wipe the mouth and nose of the neonate with sterile gauze. & 25.3 \\
\hline 2 & Dry the baby of excessive moisture. & 69.3 \\
\hline 3 & Remove wet cloth and wrap baby in warm dry cloth. & 21.3 \\
\hline 4 & Provide warmth and put baby under radiant heater. & 97.3 \\
\hline 5 & $\begin{array}{l}\text { Place the baby on flat surface and position the head of the baby so that the neck is slightly extended to open the } \\
\text { airway. }\end{array}$ & 66.7 \\
\hline 6 & Quickly clear airway. & 80.0 \\
\hline 7 & Reassess the baby after suctioning in order of importance; respirations, heart rate and color. & 80.0 \\
\hline 8 & $\begin{array}{l}\text { If the baby is not breathing, apneic or has gasping respirations or heart rate of less than } 100 \mathrm{~b} / \mathrm{min} \text {, start } \\
\text { ventilating with bag and mask. }\end{array}$ & 34.7 \\
\hline 9 & Recheck the newborns neck if it is slightly extended. & 66.7 \\
\hline 10 & $\begin{array}{l}\text { Select appropriate mask size to cover the chin, mouth and nose and check that the mask is properly sealed over } \\
\text { the newborn's nose and mouth. }\end{array}$ & 38.0 \\
\hline 11 & $\begin{array}{l}\text { Once the seal is assured and chest movement is present ventilate the newborn and maintain correct rate } \\
\text { ( } 40 \text { breaths per minute). }\end{array}$ & 71.0 \\
\hline 12 & If chest is not rising, repeat suctioning of the mouth, nose and remove mucus or blood from the airway. & 25.0 \\
\hline 13 & After 30 seconds of proper ventilation, re-evaluate breathing, heart rate and color. & 27.0 \\
\hline 14 & After ventilating for 1 minute, stop and quickly assess if newborn is having spontaneous breathing. & 21.0 \\
\hline 15 & $\begin{array}{l}\text { If the newborn is not breathing or breathing is slow ( }<30 \text { breaths per minute) or weak or severe in drawing } \\
\text { continue ventilating until spontaneous breathing begins. }\end{array}$ & 15.0 \\
\hline 16 & If newborn starts crying, stop ventilating and continue observing breathing for 15 minutes. & 12.0 \\
\hline 17 & If there is severe in drawing of the chest, ventilate with oxygen if available. & 62.0 \\
\hline 18 & $\begin{array}{l}\text { If the newborn is not breathing regularly after } 20 \text { minutes of ventilation incubate and transfer the baby for } \\
\text { appropriate care for sick new born. }\end{array}$ & 34.7 \\
\hline 19 & $\begin{array}{l}\text { If breathing is normal ( } 30 \text { - } 60 \text { breaths per minute) and there is no in-drawing or granting and no further } \\
\text { resuscitation is needed, proceed with care of the new born. }\end{array}$ & 12.0 \\
\hline
\end{tabular}

wives of different cadres and skills. The skill mix is important for comprehensive delivery of maternal and neonatal care as lower midwives are able to refer to the next level within the same department. Professional midwives have the necessary training and skills to deliver essential maternal and neonatal care, including neonatal resuscitation and timely management of birth asphyxia [8]. The Chatinkha maternity was patronized by mostly young midwives who also had less experience in the maternity ward, thus compromising quality management of pregnant women and neonates. Midwives require relevant experience to effectively implement the newborn resuscitation guidelines [7].

Results on midwives comprehension of birth asphyxia revealed both strengths and weaknesses. Overall findings indicated that most midwives had knowledge of several aspects of birth asphyxia. These results may be due to the fact that most of them had attended in-service courses in which birth asphyxia was taught. Training of health workers in obstetric emergencies improves neonatal care and outcome [9]. Thus the midwives were conversant with signs of birth asphyxia as also reported earlier at the same health facility [10] probably due to the coverage of the in-service curriculum on this aspect. The results that some midwives were still not conversant with some aspects of birth asphyxia pose a concern in a country where neonatal mortality is as high as $22 \%$ [4]. Early detection of asphyxia is critical for timely resuscitation because it is an obstetric emergency that requires timely intervention. Midwives who did not attend any in service training recently on birth asphyxia were less likely to recognize asphyxiated babies early [6]. To improve neonatal care, there is therefore a need for ongoing in-service training for midwives to understand birth asphyxia and offer its effective and timely management.

\subsection{Midwives Ability to Identify Warning Signs of Birth Asphyxia}

Overall, the findings indicated that midwives ability to identify warning signs of birth asphyxia was substandard in most of the parameters measured as per partograph review. The fetal heart rate for example was not monitored according to the guidelines. Instead of checking the 
fetal heart half hourly, it was monitored every 2 hours or was not monitored at all as also reported from Mozambique [11] in which the fetal heart was not monitored on half of the partographs despite having the risk factor of asphyxia. Color of liquor which is supposed to be monitored together with fetal heart to assess the fetal condition was also not done according to the guidelines. Furthermore, moulding and caput which were supposed to be monitored at every vaginal examination, i.e. every 4 hours was also not checked as required. Instead, it was either checked once in first stage of labor or not at all. These results agree with those reported from Sweden [12] that midwives neglected the monitoring of fetal well being in $98 \%$ of the cases. Negligence in the monitoring of the risk factors for birth asphyxia can lead to missing asphyxiated babies by as high margin as 71\% [12].

The results show that the biggest problem among the participants in this study was to monitor women for asphyxia risk factors using the partographs. If properly utilized, partographs serve as a tool for early identification of prolonged labor or as a guide to identify warning signs of birth asphyxia. Negligence in the use of partographs or their improper use puts a baby at increased risk of birth asphyxia as it becomes difficult to detect if the baby is distressed. According to Singhal \& Bhuta [13], more than half of newborns requiring resuscitation could be identified before birth if the mother is monitored appropriately for risk factors. Similar results were reported by Christensson et al. [11] in Mozambique where partographs were used to monitor only half of the deliveries. The results show that young and less experienced midwives had problems with partograph use. In addition, shortage of midwives at the ward might have contributed to improper partograph use as on average there were only 10 to 12 midwives on duty in 2 labor wards that had 28 labor beds. It was therefore practically impossible to properly monitor all the laboring women. Similar results were reported by Lang, et al. [14] and Stanton \& Rutherford [15] that heavy nursing work loads adversely affected patient safety. Results show that some at risk babies who needed resuscitation at birth were not detected before birth due to failure of the midwives to recognize the warning signs. Therefore, there is a need to continuously train midwives through in service training on resuscitation procedures for babies with birth asphyxia.

Results that more than half of the midwives failed to Apgar score using Virginia Apgar Scoring charts had implication on quality of care at the ward. Participants failed to score despite the fact that the scoring charts were readily available in the labor ward cubicles. Instead the midwives gave Apgar scores to the neonates using their experiences as also reported by Christtensson, et al. [11]. Scoring babies based on experience is not a recommended practice, hence a risk factor because the ba- bies might have been given a wrong Apgar score that compromised the quality of care. There is therefore a need to re-orient midwives in using Virginia Apgar scoring charts appropriately and correctly.

Despite the shortfalls in their practice, all midwives were able to identify the neonates that required resuscitation, probably due to the in-service training they received. However they knew after the neonate was already born and after some time had elapsed. It would be appropriate for the midwives to identify warning signs of birth asphyxia early, i.e., before the neonate is born so that they provide timely intervention to the baby immediately after birth. The warning signs of birth asphyxia are detected before birth when partographs are properly used [7]. Enforcement of partograph use by midwives on all women in labor is critical to optimize positive neonatal outcome in the detection of birth asphyxia because it serves as a tool for early detection and timely intervention to prevent or manage birth asphyxia. There is therefore a need for midwife supervisors to ensure that midwives use partograph on all women that present themselves at the maternity.

\subsection{Midwives' Adherence to Resuscitation Guidelines}

Results on midwives' adherence to resuscitation guidelines show that the procedure on the neonates who needed it was done below the recommended standards. Midwives scored over $50 \%$ in only 8 out of 19 resuscitation steps. Midwives adhered to resuscitation steps that concerned prevention of heat loss and obstruction of airway. Similar results were reported by Christtensson, et al. [11] from Mozambique and Berglund, et al. [12] from Sweden. However, most of the crucial steps that lead to neonate prolonged exposure to moisture were not adhered to. These steps are crucial because if not properly done, the neonate develops hypothermia and metabolic problems due to cold stress [16]. In addition, cold stress worsens the condition of the already asphyxiated baby due to high oxygen consumption. Some of the steps like selecting appropriate mask size to cover the chin, mouth and nose and check if the mask is properly sealed over the newborn's nose and mouth were also not adhered to. Furthermore, some of the well fitted masks leaked out air, indicating shortcomings in the prior equipment preparation. Consequently, most neonates did not get adequate ventilation due to either improper mask fitting or leaking of the ambu bags. Quality of asphyxiated babies was further compromised when the neonates that met the criteria to be put on mask were not. Similar to the finding in this study, Berglund et al., [12] reported that artificial ventilation was not initiated within one minute after birth in seven infants despite clear indication of the need for ventilation. Timely initiation of ventilation is important 
to prevent neonatal brain damage and remove mechanical obstruction from blood, meconium or mucus.

Results further show that the neonates were not reevaluated for proper breathing, color and heart rate at 30 seconds and 1 minute respectively. This step is important because these are the most important indicators for resuscitation efficacy. Continued ventilation of the neonates when not breathing, or having slow breathing was also not well adhered to because when the neonate had started a feeble cry, a midwife just commenced the neonate on oxygen and rushed back to the mother. There was therefore lack of continuous observation of the neonates when the newborn had started crying. Similar results are reported by Bream, et al. [10] and Etsy, [17] that midwives' priority was to serve the mother and not the neonate. The major cause for insufficient attention being given to the neonate is the staff shortage and heavy nurse workload in the maternity. The results therefore show a need for the health facility to deploy separate midwives for the neonates and the mothers. However, the deployment of nurses specifically for the mother and neonate is not usually done due to the shortage of staff. Therefore there is a need to train and recruit more nurses for all the health facilities of the country to improve both the maternal and neonatal care.

Transfer of the neonates to the nursery for appropriate care was done on only few babies. Neonates who have peripheral compromise and undergone resuscitation needed to be observed in a neonatal nursery for heart rate and blood pressure [18]. Thus, these observations were done only on few neonates. It was also observed that those neonates that required intubation did not receive the service due to inadequate skills by midwives. Furthermore, immediate care of the newborns that had stabilized was only given to very few babies. Immediate care of the newborn is critical in the prevention of neonatal mortality because babies with perinatal compromise and have undergone resuscitation may later manifest with HIE [19]. It is therefore important that the babies are observed to detect complications early. In Pakistan, [19] health care workers performed poorly in resuscitation skills. Hence, a lot of neonates did not get adequate resuscitation which contributed to negative neonatal outcomes. In this study, despite some challenges that the midwives encountered such as staff shortage and in adequate equipment, staff negative attitude also played a role. There were instances where the midwives were able to perform according to standard but they did not due to their negative attitude towards their work and patients. Thus with the current staffing levels the midwives could have performed better than they did if they had positive attitudes towards their work. Therefore there is a need for intensive supervision of staff in the maternity by the senior officers to ensure that the few nurses that are available are able to adhere to the set professional standards. Adherence to resuscitation guidelines is critical to the attainment of MDG 4 as birth asphyxia contributes to Malawi's high neonatal mortality rates.

Shortage of resources, equipment also contributed to midwives' non adherence to resuscitation guidelines. At times the midwife knew what to do but limited equipment and supplies hindered them from providing standard quality of care. The maternity department had two labor wards, paying and non paying wards. In both labor wards only a few resuscitation equipment were functioning at the time of the study and supplies were not available for most of the equipment. In the paying ward, the resuscitaire was not available hence the staff depended upon the few that were functioning in the non paying wards. Recently, Newton and English [20] reviewed the evidence for neonatal resuscitation and concluded that effective resuscitation was possible with basic equipment and skills in low-resource settings. Training providers in neonatal resuscitation in health facilities may prevent $30 \%$ of deaths of full-term babies with intrapartum-related events, as well as $5 \%-10 \%$ of deaths due to preterm birth [21]. Therefore, universal application of basic resuscitation may save hundreds of thousands of newborn lives currently lost each year, and contribute significantly to progress toward MDG 4. To achieve impact, the challenge is to improve obstetric care and provide universal coverage of basic resuscitation where resources, including equipment and supplies are limited. Therefore there is a need for the health facility to repair and maintain the resuscitation equipment and ensure availability of supplies so that they are available whenever the need arises.

\subsection{Limitation of the Study}

In this study, data was collected from only one facility and the findings may not be representative of the whole Malawi due to lack of representation. The training materials for in-service courses that were offered to midwives by the Ministry of health were not evaluated to identify the gaps in the training curriculum. The fact that the study was conducted by fellow midwives might have also influenced the results.

\section{CONCLUSION}

Early detection, prevention and proper management of birth asphyxia is critical in the reduction of neonatal mortality in Malawi. Though midwives in Malawi have knowledge on most aspects of birth asphyxia, there are still problems among them in the utilization of the partograph to identify the warning signs of birth asphyxia. Consequently, the midwives do not prepare in advance to prevent and provide timely and quality care when it oc- 
curs. Midwives performed well in only a few steps that dealt with prevention of heat loss and obstruction of the airway but missed out on most critical life saving steps. The non adherence to resuscitation guidelines coupled with shortage of staff and bad staff attitude towards their work is therefore contributing to high neonatal mortality rates in Malawi. There is therefore a need to strengthen the use of partographs to monitor pregnant women during labor and close supervision of maternity staff. In addition, there is need for continuous training of providers in resuscitation and partograph use. There is also a need for the health facility to ensure availability essential resuscitation equipment, supplies and resources to provide quality neonatal care thereby reducing the high neonatal mortality rate.

\section{ACKNOWLEDGEMENTS}

The study was conducted as part of the senior author's Master of Science degree in Midwifery at the University of Malawi, Kamuzu College of Nursing with a scholarship from USAID. The preparation of the manuscripts for publication was funded by the University of Tromso, Norway and the Agency for Norwegian Development Cooperation.

\section{REFERENCES}

[1] Lawn, J.E., Cousens, S. and Zupan, J. (2009) Neonatal Survival Steering Team: 4 Million Neonatal Deaths: When? Where? Why? Lancet, 365, 891-900.

[2] Davis, P.G., O’Donnell, C.P.F., Tan, A. and Schulze, A. (2004) Air versus oxygen for resuscitation of infants at birth. The Cochrane Database of Systematic Reviews, 2, CD002273.

[3] National Statistics Office (NSO) and ICF Macro (2011) Malawi demographic and health survey. Calverton, Maryland.

[4] MICS (Malawi Multiple Indicator Cluster Survey) (2006) Preliminary report. National Statistical Office, Zomba.

[5] WHO (2009) Monitoring emergency obstetric care: A handbook: Averting maternal death and disability. World Health Organization, Geneva.

[6] Ministry of Health (2010) Malawi 2010 EMONC needs assessment final report. Malawi Ministry of Health, Lilongwe.

[7] World Health Organization (2005) Integrated management of pregnancy and childbirth: A guide for midwives and doctors. World Health Organization, Geneva.

[8] Kenzie, B. and Gomez, P. (2004) Basic maternal and neonatal care: A guide for skilled providers. The access program, USAID/JHPIEGO, Baltimore. http://www.BMNCrevmanEN.pdf

[9] Draycott, T., Sibanda, T., Owen, L., Akand, V., Winter, C. and Reading, S. (2006) Does training in obstetric emergencies improve neonatal outcome? BJOG: An International Journal of Obstetrics \& Gynaecology, 113, 177182. doi:10.1111/j.1471-0528.2006.00800.x
[10] Bream, K.D., Gennero, S., Kafulafula, U. Mbweza, E. and David, H. (2005) Barriers to and facilitators for newborn resuscitation in Malawi, African. Journal of Midwifery and Women's Health, 50, 329-334. doi:10.1016/j.jmwh.2005.04.006

[11] Christensson, K., Petterson, K.O., Bulgalho, A., Manuela, C.M., Dgedge, C., Johansson, E. and Belgstrom, S. (2006) The challenge of improving perinatal care with limited resources. Observations of midwifery practices in Mozambique. African Journal of Reproductive Health, 10, 47-61. doi:10.2307/30032443

[12] Berglund, S., Grunewald, C., Pettersson, H. and Cnattingius, S. (2010) Risk factors associated with substandard care during labour. Acta Obstetrica et Gynecological Scandinavia, 89, 39-48. doi:10.3109/00016340903418751

[13] Singhal, N. and Bhuttta, Z.A. (2008). Newborn resuscitation in resource-limited settings. Seminars in Fetal \& Neonatal Medicine, 13, 432-439. doi:10.1016/j.siny.2008.04.013

[14] Lang, T.A., Hodge, M. and Olson, V. (2004) Nurse-patient ratios: A systematic review on the effects of nurse staffing on patient, nurse employee, and hospital outcomes. Journal of Nursing Administration, 34, 326-337. doi:10.1097/00005110-200407000-00005

[15] Stanton, M.W. and Rutherford, M.K. (2004) Hospital nurse staffing and quality care: Rockvilla, MD agency for health care research and quality, 2004. http://www.ahrq.gov/research/nursestaff/nursestaff.pdf

[16] Shrestha, M., Singh, R. and Upreti, D. (2009) Quality of care provided to newborns by nursing personnel at BP Koirala Institute of Health Sciences. Kathmandu University Medical Journal, 7, 231-237.

[17] Etsey, P.M. (2008) Towards the millennium development goal 5: Assessing the quality of supervised delivery in the G. A. West District, Ghana. M.Sc. Thesis, University of Ghana, Acra.

[18] Braner, D.A.V., Denson, S.E. and Ibsen, L.M. (2000) Textbook for neonatal resuscitation. 4th Edition, American Heart Association, Dallas.

[19] Ariff, S., Soofi, S., Sadik, K., Feroze, A., Khan, S. and Jafarey, S. (2010) Evaluation of health workforce competence in maternal and neonatal issues in public health sector of Pakistan: An assessment of their training needs. BMC Health Services Research, 10, 319. doi:10.1186/1472-6963-10-319

[20] Newton, O. and English, M. (2006) Newborn resuscitation: Defining best practice for low-income settings. Transactions of the Royal Society of Tropical Medicine and Hygiene, 100, 899-908. doi:10.1016/j.trstmh.2006.02.012

[21] Bhutta, Z.A., Ahmed, T., Black, R.E., Cousens, S., Dewey, K. and Giugliani, E. (2008) What works? Interventions for maternal and child undernutrition and survival. Lancet, 371, 417-440. doi:10.1016/S0140-6736(07)61693-6 